\title{
A HYBRID MESH HARMONIC BALANCE SOLVER FOR THE AEROELASTIC ANALYSIS OF TURBOMACHINERY
}

\author{
Hans-Peter Kersken*, Graham Ashcroft, Christian Frey \\ German Aerospace Center (DLR) \\ Institute of Propulsion Technology \\ Linder Höhe, 51147 Cologne, Germany \\ email:\{hans-peter.kersken, graham.ashcroft, christian.frey\}@dlr.de
}

Keywords: CFD, Harmonic Balance Method, Unstructured Grid, Flutter, Aeroelasticity, Turbomachinery

\begin{abstract}
An implementation of a Harmonic Balance method to simulate time-periodic, nonlinear flows in turbomachinery on hybrid grids comprising both structured and unstructured blocks is described. It has been developed with the aim to apply it to aeroelastic analysis problems which require support for deforming meshes. The necessary modifications of an existing implementation of a Harmonic Balance method on structured grids are presented. Especially block boundaries require a separate treatment. For the same spatial discretization order the solver for unstructured grids requires a moderate overhead in computational resources in terms of computation time compared to the structured solver when applied on grids with similar spatial resolution. The extended $H B$ solver is applied to a standard turbomachinery aeroelastic test case for inviscid flows including both subsonic and transonic configurations. The computational results obtained are compared with results from a Harmonic Balance solver for structured grids and references from literature.
\end{abstract}




\section{INTRODUCTION}

Harmonic Balance (HB) methods have been introduced into turbomachinery CFD to efficiently simulate periodic time-dependent flow phenomena by taking into account frequencies relevant for the phenomenon under investigation only. These phenomena have traditionally been simulated based on linearized governing equations starting from two-dimensional Euler equations to finally three-dimensional compressible Navier-Stokes equations. The linearization decouples the governing equations for the flow perturbations from those governing the mean flow and allows highly efficient solvers to be constructed for the perturbations. Whilst highly efficient this approach does not take into account nonlinear phenomena. Therefore such tools are not expected to give accurate results when applied to problems in which nonlinear effects are suspected to play an important role. HB methods, see for example [1, 2, 3, 4], overcome these restrictions and allow frequency-domain methods to be applied to nonlinear, time-periodic problems thereby taking into account the nonlinear coupling of perturbations and the mean flow.

The extension of the Harmonic Balance solver to unstructured and finally hybrid grids, comprising both structured and unstructured blocks, is driven by the requirement to include geometrically complex components of turbomachines like cooling channels or cavities, which are often modelled by unstructured grids. These geometrical details are a source of disturbances which may excite blade vibrations and therefore have to be taken into account when investigating aeroelastic phenomena. The newly developed solver for unstructured grids is based on previous work on aeroelastic analysis on unstructured grids with a time-linearized solver [5] and the development of a Harmonic Balance method on deforming grids [6] in the framework of DLR's block structured compressible URANS solver TRACE [7].

This paper is organized as follows: first an overview of the base flow solver, the HB method and the spatial discretization schemes is given. Then the modifications to the implementation are explained which are necessary when extending the existing HB implementation to unstructured grids. Finally results are compared to reference results when the solver for unstructured grids is applied to a duct and the Standard Configuration 10 aeroelastic test case.

We will restrict ourselves in this paper to inviscid flows as the initial validation step for the HB solver on hybrid grids.

\section{NUMERICAL METHOD}

\subsection{Underlying flow solver}

TRACE [7] is a parallel Navier-Stokes flow solver for structured and unstructured grids that has been developed at DLR's Institute of Propulsion Technology to model and investigate turbomachinery flows. The code solves the finite-volume discretization of the compressible Reynolds-averaged Navier-Stokes (RANS) equations in the relative frame of reference using a multi-block approach. For details of the discretization on structured grid refer to [8] and for a detailed description of the unstructured discretization see [9]. For the present work it is sufficient to note that following the discretization of the spatial operators in the Navier-Stokes equations the following system of ordinary differential equations (ODEs) is obtained

$$
\frac{d \mathbf{q}}{d t}+\mathbf{R}(\mathbf{q}(t))=0
$$

where $\mathbf{q}$ is the vector of conservative variables, $\mathbf{R}$ is the discretized RANS residual vector and $t$ denotes the physical time. 


\subsection{Harmonic balance method}

The Harmonic Balance method described in this work is part of the CFD code TRACE. For completeness we will summarize the HB algorithm as it is implemented in TRACE. For a detailed description see [10]. Time-periodic solutions of Eqn. (1) can often be described by a limited number of solution harmonics, i.e.,

$$
\mathbf{q}(x, t)=\operatorname{Re}\left[\sum_{k=0}^{K} \hat{\mathbf{q}}_{k}(x) e^{i k \omega t}\right]
$$

where $\hat{\mathbf{q}}_{k}$ are the complex valued solution harmonics and $\omega=2 \pi f$ is the fundamental angular frequency. In such cases it is computationally attractive to formulate the unsteady problem, Eqn. (1), in the frequency domain, i.e. to consider

$$
\mathbf{i} k \omega \hat{\mathbf{q}}_{k}+\widehat{\mathbf{R}(\mathbf{q})_{k}}=0
$$

for only a finite number of harmonics, $k=0, \ldots, K$. For configurations in which non-linearity can be assumed to be negligible the coupling between the harmonics of $\mathbf{q}$, imposed by the nonlinear nature of $\mathbf{R}$, can be neglected and one obtains $K+1$ independent equations for the solution harmonics and the time-mean solution field. Here the equations for the harmonics are linear and only for the time-mean solution has a nonlinear equation to be solved. However, if one wishes to retain nonlinear effects an alternative approach is required in the modelling or computation of $\widehat{\mathbf{R}(\mathbf{q})}_{k}$. We compute $\widehat{\mathbf{R}(\mathbf{q})}_{k}$ as $\left.\mathscr{F}(\mathbf{R}(\mathscr{F}-1 \hat{\mathbf{q}}))\right|_{k}$ and therefore solve

$$
\mathbf{i} k \omega \hat{\mathbf{q}}_{k}+\left.\mathscr{F}\left(\mathbf{R}\left(\mathscr{F}^{-1} \hat{\mathbf{q}}\right)\right)\right|_{k}=0,
$$

where $\mathscr{F}$ denotes the Discrete Fourier Transform (DFT) and $\mathscr{F}^{-1}$ its inverse. Eqn. (4) is solved in the frequency domain to obtain the complex valued harmonics of the conservative variables $\hat{\mathbf{q}}_{k}$. To compute the harmonics of the RANS residual vector $\mathbf{R}$ the solution field vector is first reconstructed at $N$ sampling points within the period of oscillation from the Fourier coefficients of the conservative variables $\hat{\mathbf{q}}_{k}$ using the inverse DFT. With the reconstructed solution vectors the RANS residual vectors $\mathbf{R}$ are then computed at the $N$ sampling points to enable the DFT of $\mathbf{R}$ to be computed, i.e., the $\widehat{\mathbf{R}(\mathbf{q})}{ }_{k}$. Note, since the RANS residual $\mathbf{R}$ is evaluated in the time-domain the standard flux and discretization schemes from the underlying nonlinear solver can be used.

As a hybrid time- and frequency-domain method the approach has the advantage, over methods formulated purely in the time- or frequency-domain, of being able to employ not only the possibly highly nonlinear time-domain flux functions (and their stabilizing numerical limiters) but also highly accurate nonreflecting boundary conditions formulated in the frequency domain [11]. Particularly in the context of aeroelasticity or aeroacoustics boundary conditions are of the utmost importance. The implementation in TRACE of these boundary conditions is described in [12].

For aeroelastic analysis the approach described above has to be modified to deal with timedependent meshes as described in [6]. The governing equations are solved by an implicit pseudo-time approach. Discretizing the pseudo-time operator using the first-order Euler backward method and then linearizing the harmonic balance residual $\mathbf{R}^{\mathrm{HB}}$ in pseudo-time the following system of equations is obtained

$$
\left[\left(\frac{1}{\Delta \tau}+\mathbf{i} k \omega\right)+\left.\frac{\partial \mathbf{R}}{\partial \mathbf{q}}\right|_{\hat{\mathbf{q}}_{0}^{(m)}}\right] \Delta \hat{\mathbf{q}}^{(m)}=-R_{k}^{\mathrm{HB}}\left(\hat{\mathbf{q}}^{(m)}\right),
$$


where $\bar{V}$ is the unperturbed cell volume and

$$
R_{k}^{\mathrm{HB}}\left(\hat{\mathbf{q}}^{(m)}\right)=\mathbf{i} k \omega \widehat{\left.(V q)\right|_{k}}+\left.\mathscr{F}\left(V \mathbf{R}\left(\mathscr{F}^{-1} \hat{\mathbf{q}}\right)\right)\right|_{k}, \quad \Delta \hat{\mathbf{q}}^{(m)}=\hat{\mathbf{q}}^{(m+1)}-\hat{\mathbf{q}}^{(m)} .
$$

Here $m$ is the pseudo-time iteration counter and $V$ the time-dependent cell volume. The coupling terms have been neglected in the left-hand side and therefore it depends directly only on the time-mean solution $\hat{\mathbf{q}}_{0}^{(m)}$. As such the residual Jacobian $\frac{\partial \mathbf{R}}{\partial \mathbf{q}}$ is identical to that employed in the steady flow solver. The linear system of equations, Eqn. (5), is solved using either the incomplete lower upper (ILU) or successive overrelaxation (SSOR) methods.

\subsection{Spatial discretization}

So far no reference to a specific discretization method has been made because the algorithm described above can be applied unaltered to structured and unstructured meshes. To understand the differences between an HB implementation for structured and unstructured grids we have to look into the spatial discretization in more detail. For both types of grids the discretized residual vector $R_{i}$ at cell $i$ computed by a finite volume method is given by

$$
\mathbf{R}_{i}=\frac{1}{V_{i}} \sum_{s \in B_{i}} \mathbf{F}_{s}(\mathbf{q})-\mathbf{S}_{i}(\mathbf{q}) .
$$

Here $V_{i}$ is the volume of the cell, $B_{i}$ the set of its faces, $\mathbf{F}_{s}$ the numerical flux across face $s$ and $\mathbf{S}_{i}$ any source term. Apart from the source term, which depends on the state of the cell itself only, the residual is the sum of convective and viscous fluxes, $\mathbf{F}_{s, \text { conv }}$ and $\mathbf{F}_{s \text {,visc }}$, respectively:

$$
\mathbf{F}_{s}(\mathbf{q})=\mathbf{F}_{s, \text { conv }}\left(\mathbf{q}_{\mathrm{L}}, \mathbf{q}_{\mathbf{R}}\right)+\mathbf{F}_{s, \text { visc }}\left((\nabla \mathbf{q})_{s}\right)
$$

where $\mathbf{F}_{s \text {,conv }}$ is computed by the Roe upwind scheme [13] extended to second order accuracy using van Leers MUSCL technique [14] while $\mathbf{F}_{s \text {,visc }}$ is discretized by a second order central scheme. The so-called left and right states, $\left(\mathbf{q}_{\mathrm{L}}\right.$ and $\left.\mathbf{q}_{\mathrm{R}}\right)$ at face $s$ which is common to cells $i$ and $i^{\prime}$ are computed with second order accurate extrapolation schemes, i.e., on structured grids,

$$
\mathbf{q}_{\mathrm{L}}=\mathbf{q}_{i}+\frac{1}{2}(\widetilde{\Delta} \mathbf{q})_{\mathrm{L}}, \quad \mathbf{q}_{\mathrm{R}}=\mathbf{q}_{i^{\prime}}-\frac{1}{2}(\widetilde{\Delta} \mathbf{q})_{\mathrm{R}},
$$

whereas, on unstructured grids,

$$
\mathbf{q}_{\mathbf{L}}=\mathbf{q}_{i}+(\widetilde{\nabla} \mathbf{q})_{\mathbf{L}} \cdot\left(x_{s}-x_{i}\right), \quad \mathbf{q}_{\mathrm{R}}=\mathbf{q}_{i^{\prime}}+(\widetilde{\nabla} \mathbf{q})_{\mathrm{R}} \cdot\left(x_{s}-x_{i^{\prime}}\right)
$$

is used. By $(\widetilde{\Delta} \mathbf{q})_{\mathrm{L} / \mathrm{R}}$ and $(\widetilde{\nabla} \mathbf{q})_{\mathrm{L} / \mathrm{R}}$ we denote the limited slopes and gradients at cell centers, respectively, see [15] and [9] for details. On unstructured grids the gradients are computed using the Green-Gauss approach.

\section{IMPLEMENTATION FOR UNSTRUCTURED AND HYBRID GRIDS}

The HB solver for structured grids has been implemented by reusing computational routines for the nonlinear residual $\mathbf{R}$ and its Jacobian $\frac{\partial \mathbf{R}}{\partial \mathbf{q}}$ including the computation of the numerical fluxes according to the previous section. As examination of Eqn. (5) and Eqn. (6) shows this implementation strategy carries over to the implementation of the $\mathrm{HB}$ solver for unstructured grids by using the respective routines from the nonlinear solver for unstructured grids.

Where different and additional implementations have to be considered is at block boundaries in a multi-block configuration. Fig. 1 depicts the situation for a two dimensional grid with the 

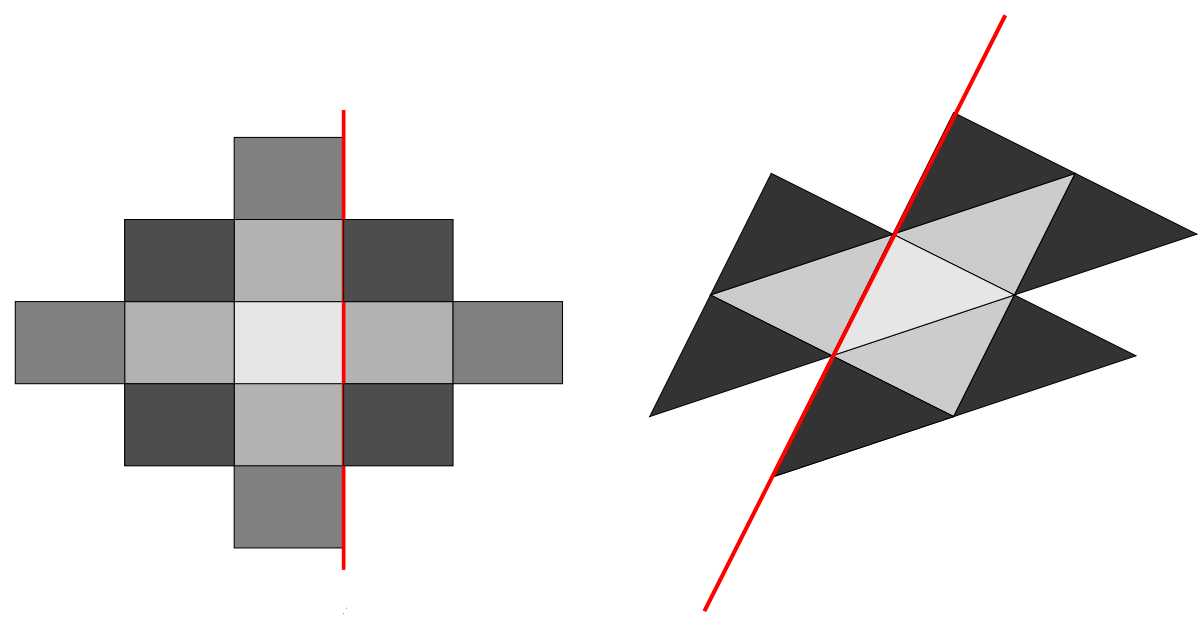

Figure 1: Stencil for the second order Navier-Stokes residual on structured and unstructured meshes [5].

focus on a single stencil where the red line marks the block boundaries. Neighbouring cells may reside on different blocks, however, their values are needed to maintain second order spatial accuracy across block boundaries, i.e., for the extrapolation given in Eqn. (9) and Eqn. (10). For completely structured grid this is solved by exchanging a two cell wide ghost cell layer surrounding each block which is filled with the respective values from neighbouring blocks. In Fig. 1 these are the cells to the right of the red line for the structured stencil. The second order extrapolation can be performed locally at block boundaries then. This procedure at block boundaries for structured grids does not carry over to unstructured grids because in TRACE we keep only one layer of ghost cells on unstructured grids which is constructed from cells having a common face with the local cell. The gradient in these cells however can not be computed locally now because information on the neighbourhood of the ghost cell, the black triangles to the left of the red line in the right picture in Fig. 1, is incomplete. Therefore in the nonlinear solver a two step process is used. First the states at the ghost cells are exchanged which allows the gradients at the cells adjacent to the boundary to be computed locally. Then the gradients are exchanged which in turn allows to interpolate gradients to boundary cell faces to compute the left and right states at the face required for second order spatial accuracy.

In the HB context we have to compute the nonlinear residual for every sampling point $t_{p}$ used to compute the harmonics of the residual $\widehat{\mathbf{R}(\mathbf{q})_{k}}=\left.\mathscr{F}\left(\mathbf{R}\left(\mathscr{F}^{-1} \hat{\mathbf{q}}\right)\right)\right|_{k}$ from the reconstructed states $\mathbf{q}\left(t_{p}\right)=\mathscr{F}^{-1} \hat{\mathbf{q}}$ and its gradients $\nabla \mathbf{q}\left(t_{p}\right)$ at the cell itself and its neighbours. If we follow the procedure for the nonlinear solver here this procedure has to be repeated for every sampling point, i.e., two communications steps are initiated each blocking the computation. It would be preferable to cluster or even avoid these communication steps. Taking the gradients at ghost cells not into account would reduce the spatial discretization order from second to first order. However, observe that the inverse DFT and the computation of the gradients are linear operations which commute, $\nabla \mathbf{q}=\nabla\left(\mathscr{F}^{-1} \hat{\mathbf{q}}\right)=\mathscr{F}^{-1}(\nabla \hat{\mathbf{q}})$. Therefore instead of computing the gradients of the states at ghost cells necessary to compute $\mathbf{R}$ at cells next to the boundary by the two step procedure they can be reconstructed from the gradients of the harmonics. This is achieved by first exchanging the harmonics $\hat{\mathbf{q}}_{k}$ and computing their gradients locally at cells which are ghost cells at neighbouring blocks. These gradients are then exchanged. Now all data are available to compute the nonlinear residual at every sampling point without further communication steps. For every sampling point first the states are reconstructed at the ghost cells and 


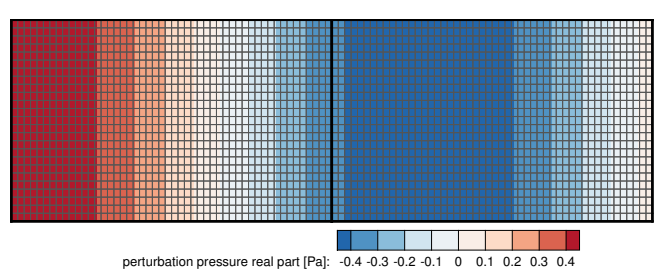

Figure 2: Grid and block topology of the 2D duct superimposed with the contours of the real part of the pressure perturbation.

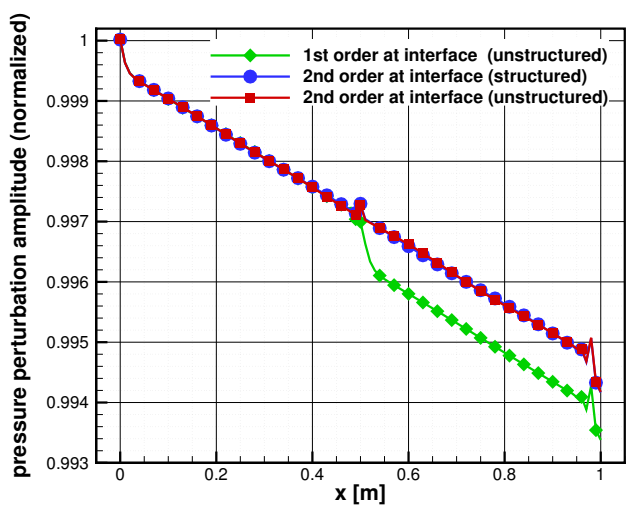

Figure 3: Normalized pressure amplitude along the center of the duct.

in the interior from their harmonics. Then the gradients at the ghost cells are reconstructed from their harmonics and finally the gradients at interior cells are computed from the reconstructed states. Now the limiter is applied and the states at the cell faces according to Eqn. (10) are computed. These steps can be performed on locally available data.

For hybrid grids comprising both structured and unstructured blocks non-matching interfaces may be generated, i.e., faces on one side of a block boundary between two blocks overlap with more than one face on the other side. In grids for turbomachinery simulation this happens especially where components with complex geometries like cooling channels or cavities are connected to the main flow geometry. Here a spatially first order coupling algorithm is implemented. The algorithm basically reconstructs the solution at the sampling points, interpolates the reconstructed states to the ghost cells at the neighbouring block and performs a DFT using all relevant frequencies at this block to update the harmonics. The algorithm is explained in detailed in [16].

\section{VALIDATION}

\subsection{Spatial discretization order at block boundaries}

To demonstrate the effectiveness of the mechanism described in section 3 a simple 2D duct configuration has been constructed comprising two blocks and 2500 cells. An acoustic wave is prescribed at the entry of the duct with an amplitude of $0.5 \mathrm{~Pa}$, a frequency of $400 \mathrm{~Hz}$ and a circumferential wave number 0 in an axial mean flow with Mach number 0.6. The grid, together with the real part of the pressure perturbation, is shown in Fig. 2. The unstructured grid is constructed from the same cells as the structured grid. In Fig. 3 the pressure perturbation amplitude is plotted along the center of the duct. Without the new implementation of the gradient computation at block boundaries a jump at the block boundary can be seen due to the degradation of the spatial discretization order. With the gradients at the ghost cells included the solution using the HB solver for unstructured grids follows the solution computed with the HB solver for structured grids. 


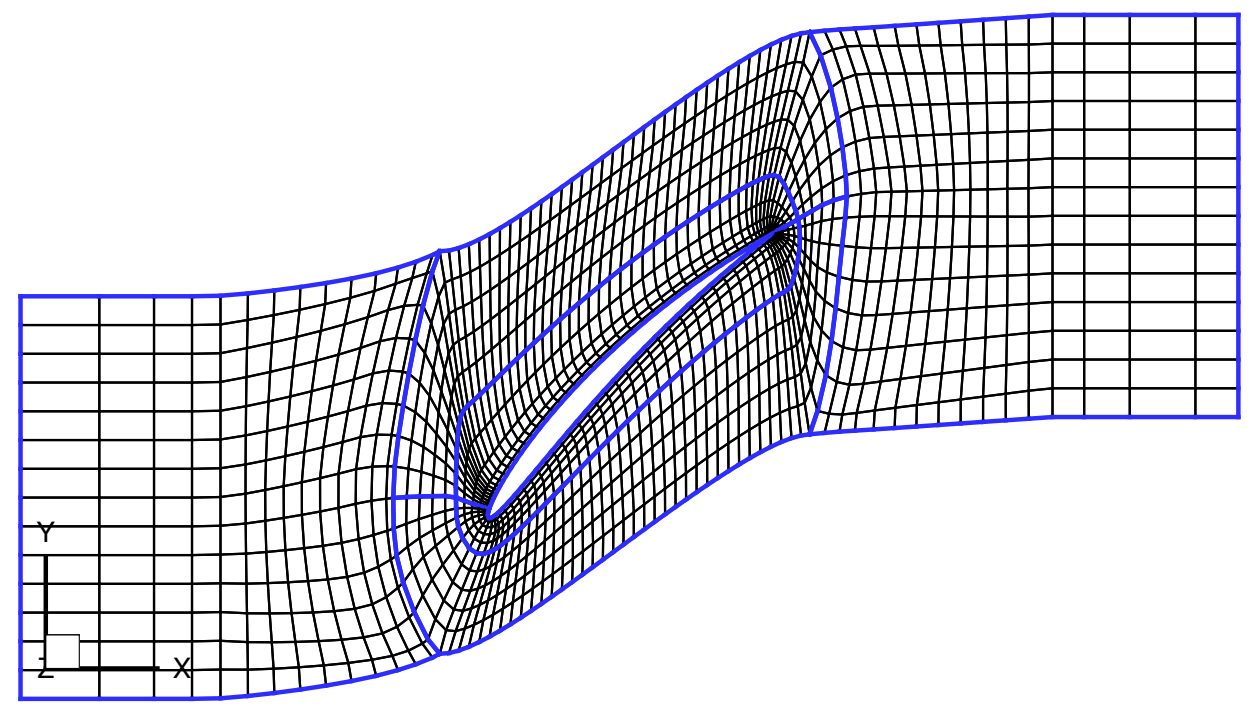

Figure 3: Computational domain, block topology and grid (every second mesh point only) for Standard Configuration 10 .

\subsection{Aeroelastic test case}

To validate the HB solver for unstructured meshes for flutter problems the extended solver is applied to the well documented aeroelastic test case Standard Configuration 10 (STCF10) [17]. The test case is simulated by solving the nonlinear Euler equations. The two-dimensional computational domain comprises a total of 5600 cells distributed over 6 blocks, see Fig. 3 . The entry and exit boundaries of the computational domain are located approximately one chord length upstream and downstream of the compressor blade, respectively. In [6] an excellent agreement of simulation result obtained with the HB solver in TRACE for block-structured grids with data from literature is reported. We will compare the results obtained with the solver for unstructured grids with these results and with results given in [18]. Fig. 4 shows the computed time-mean flow fields in terms of the isentropic Mach number $M_{i s}$ along the surface of the blade. At both operating points the numerical results agree very well with the corresponding data for the solver on structured grids. The aeroelastic stability of the two-dimensional compressor cascade
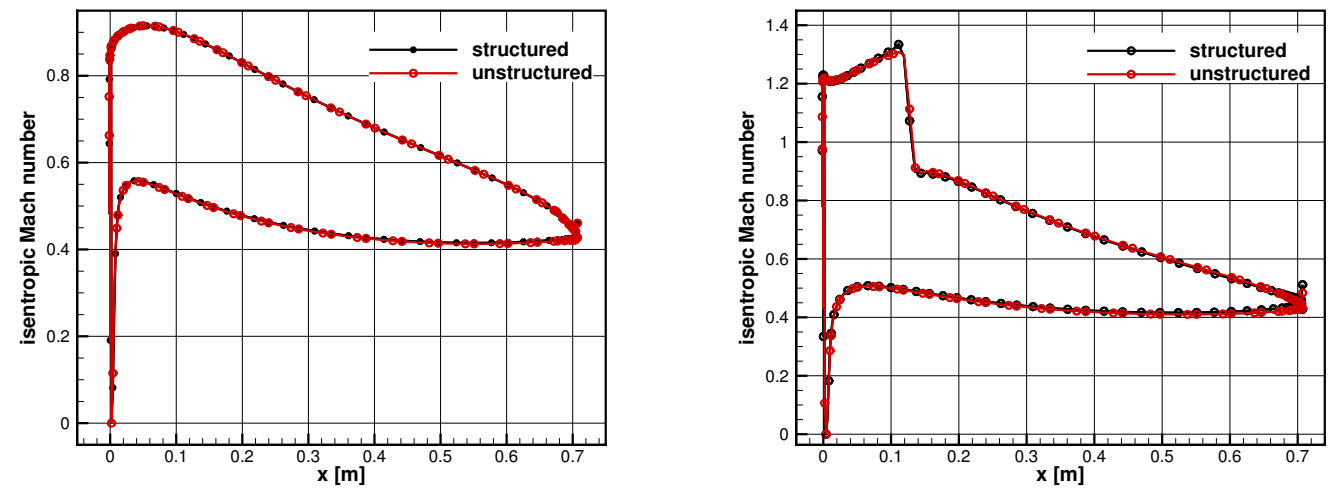

Figure 4: Isentropic Mach number distributions for the subsonic (left) and transonic cases (right) computed with the structured and the unstructured solvers. 

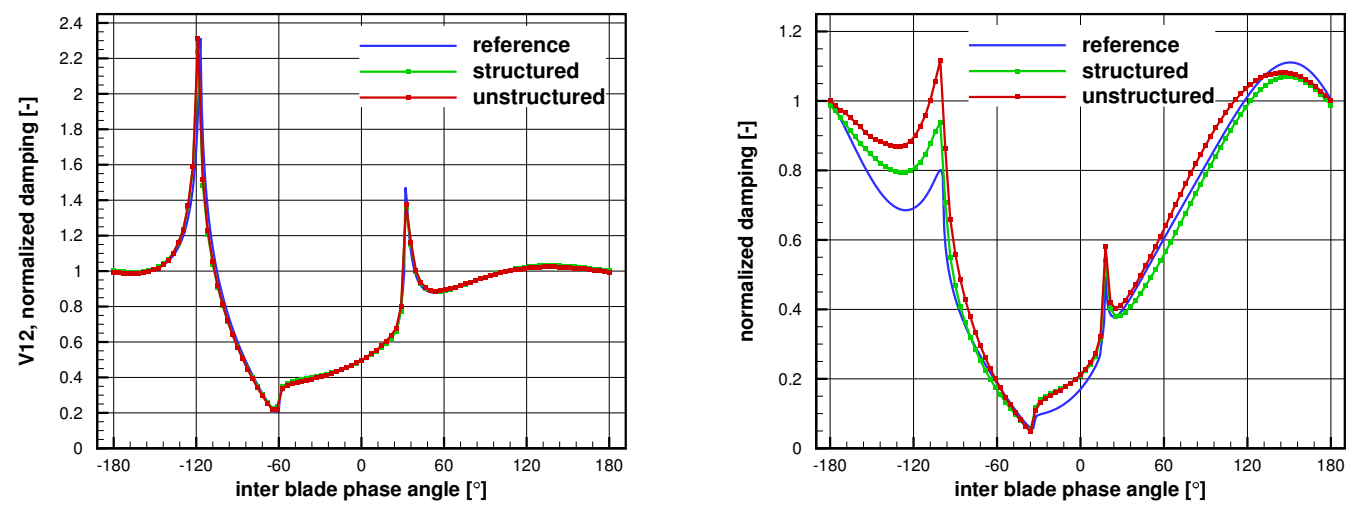

Figure 5: Normalized aerodynamic damping at subsonic (left) and transonic (right) flow conditions with $\omega^{*}=1$ and $\omega^{*}=0.5$, respectively.

is investigated for a torsional mode with an amplitude of $0.01^{\circ}$ and axis of rotation located at midchord for a reduced frequency $\omega^{*}$ of 1.0 for the subsonic and 0.5 for the transonic case. $\omega^{*}$ is defined by $\omega^{*}=c \omega /|U|_{\text {Inlet }}$. Where $c$ is the chord length and $|U|_{\text {Inlet }}$ the velocity magnitude at the inlet. The simulation has been performed with a single higher harmonic, the prescribed frequency of the torsional mode. The computed values of aerodynamic damping, normalized against the value at an inter blade phase angle of $180^{\circ}$, are shown in Fig. 5 and compared against results obtained with the HB solver for structured grids and a reference from literature [18]. As can be seen in Fig. 5 the HB solutions show very good agreement with the structured solver results and the literature results for the subsonic case. The discrepancies observed for the transonic case can be attributed to the sensitivity of the position of the acoustic resonance at an inter blade phase angel of about $-100^{\circ}$. Its position is very sensitive to the velocities at the exit boundary which in turn are sensitive to the position of the shock which depends to some extent on the specific solver parameters and the spatial resolution of the grid.

Structured and unstructured grids have the same size. Therefore we use this case to asses the overhead incurred by using the unstructured formulation instead of the structured. Fig. 6 shows the $\mathrm{L}_{1}$ residual norm of the first harmonic for an inter blade phase angel of $60^{\circ}$. Having almost the same algorithmic convergence rate as seen in the right plot of the figure, from the left plot one deduces that the computation time to achieve the same residual norm about $15 \%$
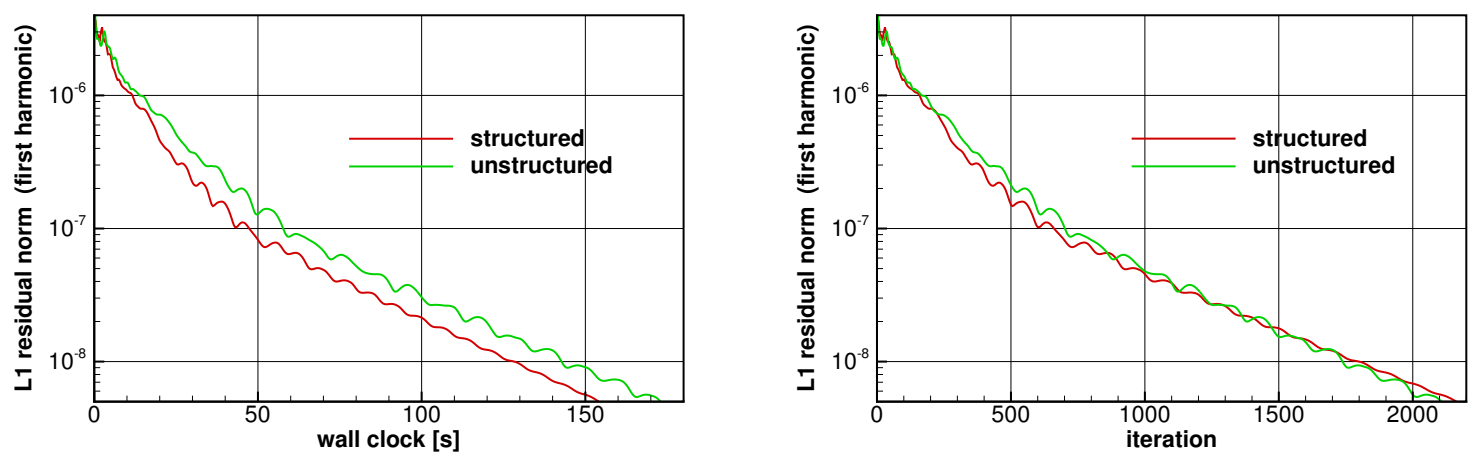

Figure 6: $\mathrm{L}_{1}$ norm of the residual for the first harmonic. 


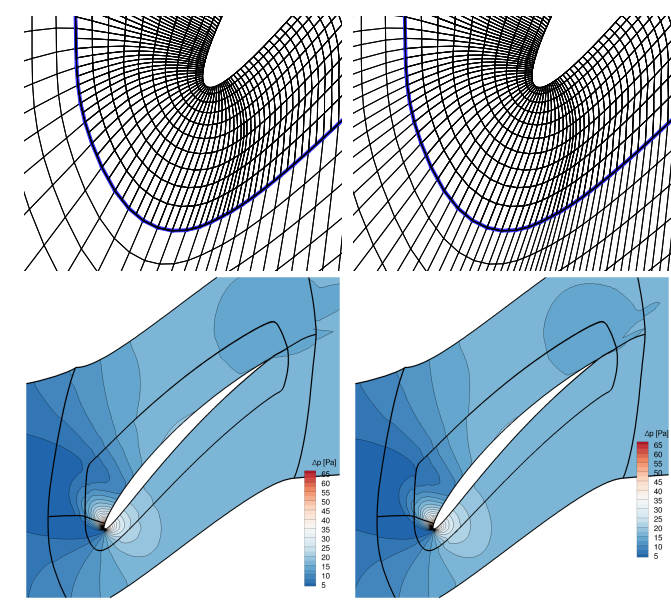

Figure 7: Mesh near the leading edge, with the interface indicated in blue, and the harmonic pressure amplitude on the hybrid grid (left) and on the refined structured grid (right).

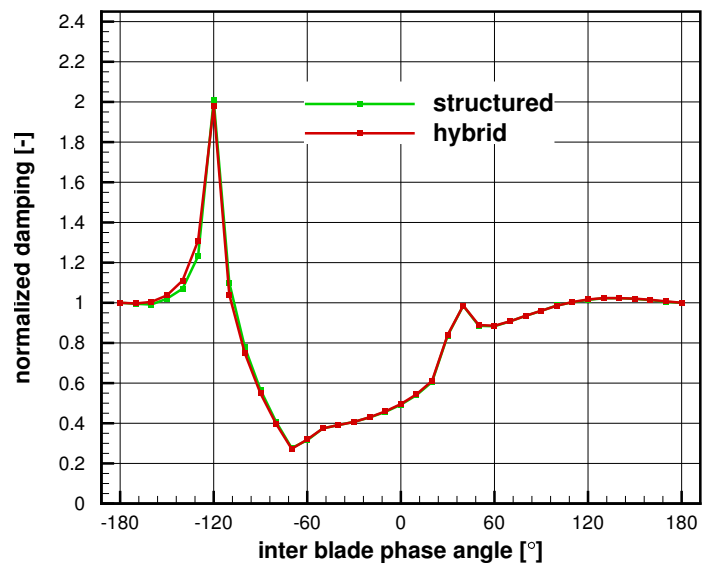

Figure 8: Damping curve normalized to value at $180^{\circ}$ computed on the refined unstructured grid and the and the hybrid grid.

larger. This is acceptable with configurations in mind where a configuration due to its complex geometry can be simulated efficiently with an unstructured grid only.

To demonstrate the ability of the solver to handle hybrid grids the O-block surrounding the blade is refined by increasing the number of grids points by a factor of two such that at the block boundary the 1-1 connectivity is replaced by a non-matching connectivity. The O-block although having a structured topology is converted to an unstructured block, see the two plots at the top of Fig. 7. Reference results have been generated on a structured grid which is refined by a factor of two globally. As shown in Fig. 7 the pressure perturbation amplitude for an inter blade phase angle of $60^{\circ}$ displays only small differences when computed on a grid with or without the non-matching interface. This holds for all other inter blade phase angles. The damping curve computed on the hybrid grid shows very good agreement with the results on the refined structured grid.

\section{SUMMARY AND OUTLOOK}

In this paper we described the steps to build a Harmonic Balance solver on hybrids grids for inviscid flows. It makes heavy use of functionality already available in the base solver. At block boundaries, both with 1-1 and non-matching connectivity, additional effort is necessary to maintain second order spatial accuracy at boundaries with 1-1 connectivities or handle the non-matching case. The new solver has been applied to the reference test case Standard Configuration 10. Very good agreement of the simulation results has been obtained with results from the HB solver for structured grids and reference results from the literature for the subsonic operation point. The differences observed for the transonic operation point can be explained by the sensitivity of the position of the acoustic resonance on the mean flow conditions. A simulation on a hybrid grid demonstrated the ability of solver to handle non-matching block boundaries.

The simulation results show that a HB method has been developed which includes the necessary ingredients for the aeroelastic analysis on hybrid grids. To apply the method to turbomachinery configurations with relevance to industry the solver will be extended to solve the URANS equations including a turbulence model by the HB method. For increased accuracy work is ongoing to maintain second order spatial discretization order at non-matching interfaces. 


\section{ACKNOWLEDGEMENTS}

This research was supported by the German Federal Ministry of Economic Affairs and Energy under grant numbers 03ET7040C as part of the AG Turbo project COOREFLEX.

\section{REFERENCES}

[1] K. C. Hall, J. P. Thomas, and W. S. Clark, "Computation of unsteady nonlinear flows in cascades using a harmonic balance technique," AIAA Journal, vol. 40, pp. 879-886, MAY 2002.

[2] K. Ekici and K. C. Hall, "Nonlinear frequency-domain analysis of unsteady flows in turbomachinery with multiple excitation frequencies," Collection of Technical Papers - AIAA Applied Aerodynamics Conference, vol. 1, pp. 623-636, 2006.

[3] A. Gopinath, E. van der Weide, J. Alonso, A. Jameson, K. Ekici, and K. Hall, "Threedimensional unsteady multi-stage turbomachinery simulations using the harmonic balance technique," in 45th AIAA Aerospace Sciences Meeting and Exhibit, Reno, NV, January 811 2007., 2007.

[4] F. Sicot, G. Dufour, and N. Gourdain, "A time-domain harmonic balance method for rotor/stator interactions," Journal of Turbomachinery, vol. 134, no. 1, p. 011001, 2012.

[5] C. Frey and H.-P. Kersken, "A hybrid mesh linear harmonic solver for the aeroelastic analysis of turbomachinery," in 6th European Conference on Computational Fluid Dynamics (ECFD VI), 2014.

[6] G. Ashcroft, C. Frey, and H.-P. Kersken, "On the development of a harmonic balance method for aeroelastic analysis," in 6th European Conference on Computational Fluid Dynamics (ECFD VI), 2014.

[7] K. Becker, K. Heitkamp, and E. Kügeler, "Recent progress in a hybrid-grid CFD solver for turbomachinery flows," in Proceedings Fifth European Conference on Computational Fluid Dynamics ECCOMAS CFD 2010, 2010.

[8] M. Franke, T. Röber, E. Kügeler, and G. Ashcroft, "Turbulence treatment in steady and unsteady turbomachinery flows," in Proceedings Fifth European Conference on Computational Fluid Dynamics ECCOMAS CFD 2010, (Lisbon, Portugal), June 2010.

[9] K. Becker and G. Ashcroft, "A comparative study of gradient reconstruction methods for unstructured meshes with application to turbomachinery flows," in 52nd AIAA Aerospace Sciences Meeting, (National Harbor, MD, USA), Jan. 2014.

[10] C. Frey, G. Ashcroft, H.-P. Kersken, and C. Voigt, "A harmonic balance technique for multistage turbomachinery applications," in Proceedings of ASME Turbo Expo 2014, 2014.

[11] M. B. Giles, "Nonreflecting boundary conditions for Euler calculations," AIAA Journal, vol. 28, no. 12, pp. 2050-2058, 1990.

[12] H.-P. Kersken, G. Ashcroft, C. Frey, N. Wolfrum, and D. Korte, "Nonreflecting boundary conditions for aeroelastic analysis in time and frequency domain 3D RANS solvers," in Proceedings of ASME Turbo Expo 2014, 2014. 
[13] P. L. Roe, “Approximate Riemann solvers, parameter vectors, and difference schemes," Journal of Computational Physics, vol. 43, no. 2, pp. 357-372, 1981.

[14] B. van Leer, "Towards the ultimate conservative difference scheme. V. a second-order sequel to Godunov's method,' Journal of Computational Physics, vol. 32, no. 1, pp. 101136, 1979.

[15] C. Hirsch, Numerical Computation of Internal and External Flows - Computational Methods for Inviscid and Viscous Flows, vol. 2. Wiley, 1 ed., 1990.

[16] C. Voigt and G. Ashcroft, "On the extension of a harmonic balance method for the simulation of compressor casing treatments," in Proceedings of the ASME Turbo Expo 2016, 2016.

[17] T. H. Fransson and J. M. Verdon, "Updated report on Standard Configurations for the Determination of unsteady Flow Through Vibrating Axial-flow Turbomachine-Cascades," Tech. Rep. TRITA/KRV/92.009, KTH, Stockholm, 1992.

[18] RPMTurbo Pty. Ltd., "RPMTurbo Standard Configuration 10.” http://rpmturbo.com/ testcases/sc10/index.html, 2005-2014. 